\title{
A Computational Study of Metal-Contacts to Beyond-Graphene 2D Semiconductor Materials
}

\author{
Jiahao Kang ${ }^{+}$, Deblina Sarkar ${ }^{+}$, Wei Liu ${ }^{+}$, Debdeep Jena ${ }^{++}$and Kaustav Banerjee ${ }^{+}$ \\ ${ }^{+}$Department of Electrical and Computer Engineering, University of California, Santa Barbara, CA 93106 \\ ${ }^{++}$Department of Electrical Engineering, University of Notre Dame, Notre Dame, IN 46556 \\ Email: \{jiahao_kang, deblina, liuwei\}@ece.ucsb.edu; djena@nd.edu; kaustav@ece.ucsb.edu
}

\begin{abstract}
Among various 2D materials, monolayer transition-metal dichalcogenides (TMDs) with intrinsic band gaps (1.1-2.2 eV) are considered as promising candidates for next generation electronics. For applicability of these novel materials as transistors, a comprehensive understanding of metal contacts to them is an absolute necessity, which is lacking at present. In this paper, we report a systematic study of metal-TMD contacts with different geometries (end-contacts and side-contacts) by ab-initio density functional theory (DFT) calculations. Particularly, contacts between $\mathrm{Au}, \mathrm{Pd}$, In or $\mathrm{Ti}$, and monolayer $\mathrm{MoS}_{2}$ or $\mathrm{WSe}_{2}$ are studied, respectively, including optimized geometries, partial density of states (PDOS), electron densities and effective potentials. Among the side-contacts to $\mathrm{MoS}_{2}$, Ti shows the potential to form the best contacts, while for $\mathrm{WSe}_{2}$ side-contacts, $\mathrm{Pd}$ exhibits the most advantages. We also find that end-contacts can be highly advantageous compared to side-contacts due to strong overlap of electron orbitals, absence of Schottky barriers and small tunnel barriers. Our modeling and simulation framework and results provide guidelines for novel $2 \mathrm{D}$ semiconductor device design and fabrication.
\end{abstract}

\section{Introduction}

As CMOS technology approaches its limits, an alternative material to Silicon is needed for future logic transistor applications. 2D semiconductors, with extremely small thicknesses (few angstroms) and pristine interfaces without out-of-plane dangling bonds, have the potential to allow efficient electrostatics, reduction of short channel effects, fewer traps on semiconductor-dielectric interface and high degree of vertical scaling. Recently, many types of 2D materials including graphene and TMDs have been experimentally and theoretically demonstrated as shown in Fig. 1 (a). While graphene [1],[2], does not have a band gap, TMDs, a family of 2D semiconductor layers arranged in hexagonal lattice (Fig. 1 (b) and (c)) exhibit considerable band gap $\left(E_{g}\right)(1.1-2.2 \mathrm{eV})$ (Fig. 1 (a) and (d)) and thus present great potential for low-power digital applications [3]-[5].

The mobility of carriers in TMD semiconductors is currently lower than in graphene although it can be boosted by deposition of high- $\kappa$ dielectrics over or below TMD materials due to suppression of the Coulomb-scattering. While use of proper work-function metal and doping can improve the extracted field-effect mobility to some extent by reducing the Schottky barrier and improving the contact [5 ], the contact resistance still remains considerably higher than desirable values. Hence, in order to achieve high performance it is necessary to understand the nature of the electronic interface between a metal and TMD layers in detail, going beyond the analytical Schottky barrier theory. However, currently such a comprehensive study of metal-TMD contact is lacking. Particularly, the properties of metal-TMD interfaces including side-contacts (Fig. 2 (a)) and especially end-contacts (Fig. 2 (b)) have not been reported in detail, except a work on $\mathrm{MoS}_{2}$ contact using DFT [8] where the study was limited to the specific cases of Ti-MoS 2 and $\mathrm{Au}-\mathrm{MoS}_{2}$ side-contacts. In this paper, we present the study of contacts between monolayer $\mathrm{MoS}_{2}$ and $\mathrm{WSe}_{2}$ and various metals $\mathrm{Au}, \mathrm{Pd}, \mathrm{In}$ and $\mathrm{Ti}$ for different contact geometries (end- and side- contacts). We show that apart from choosing a proper work-function metal, the detailed physics of the interface between the metal and the TMD layers plays an important role, which should be understood to achieve low contact resistances.

\section{Methodology}

The computational study of metal-TMD contacts is performed in four steps as listed in Fig. 2 (d). Although we study $\mathrm{MoS}_{2}$ and $\mathrm{WSe}_{2}$ as examples, the methodology can be applied to a broad range of 2D semiconductors. The first three steps are:

Choosing Metals: Considering fundamental physical properties (melting point, electrical and thermal conductance) as well as chemical properties (stability and toxicity) of all metals, Al, Ti, Cr, $\mathrm{Ni}, \mathrm{Cu}, \mathrm{Pd}, \mathrm{Ag}, \mathrm{In}, \mathrm{Pt}$ and $\mathrm{Au}$ are usually suitable as contact metals. However, the contact metals for TMDs should either have low work functions (WFs) to achieve small n-type Schottky barriers (SBs) or have high WFs to achieve small p-type SBs. Ag and $\mathrm{Cr}$ can be excluded because of their unsuitable WFs with respect to the TMDs [9]. Furthermore, $\mathrm{Cr}$ and $\mathrm{Ni}$ can also be excluded due to large lattice mismatches (defined in [10]) with TMD, because small lattice mismatches are required to maximize orbital overlaps [8]. $\mathrm{Al}$ is not a good contact metal for TMD because of absence of d-orbitals, which can mix with Mo and $\mathrm{W}$ d-orbitals resulting in better electron injection [8]. In addition, our experimental results have shown that $\mathrm{Al}$ and Ni cannot form low-resistance contacts with TMD [11].

Based on the above criteria, $\mathrm{Au}, \mathrm{Pd}$, In and Ti are chosen as the contact metals for this study. It should be noted that $\mathrm{Au}$ and $\mathrm{Pd}$ are high WF metals with very similar WFs and may form p-type contacts with TMDs, while In and Ti have very similar low WFs leading to n-type contacts (Fig. 1 (a)).

Interface Modeling: As shown in Fig. 3 and Fig. 4, metal-TMD contact regions are simulated, which are periodic in the $x$ and $y$ directions (Fig. 3 (j)) and separated by vacuum in the $z$ direction (Fig. 3 (i)). For side-contacts, the contact region contains an intrinsic TMD monolayer and the close-packed surfaces of a metal $(\mathrm{Au}(111)$, $\operatorname{Pd}(111), \operatorname{In}(101)[12]$ or $\operatorname{Ti}(001))$ extending to the $6^{\text {th }}$ layer. In this work, to emulate the effect of upper layers in modeling, the $3^{\text {rd }}$ to $6^{\text {th }}$ layers of metal from the interface are set as constraints (atoms with fixed locations) as shown in Fig. 3 (a) and (h). TMD as well as $1^{\text {st }}$ to $2^{\text {nd }}$ metal layers are left to relax. Although in real situations, the contact metals consist of many layers, we restrict the simulation to 6 layers because the obtained results do not change appreciably beyond this thickness (Fig. 3 (I)).

For end-contacts, as shown in Fig. 4 (a), the several TMD unit cells on the left are constraints to emulate the effect of a long TMD layer on the left (same as the configuration for metal-graphene endcontacts [13]). All other atoms are allowed to relax.

DFT Calculations: The numerical DFT calculations were performed using Atomistix ToolKit (ATK) [14]. Both PerdewBurke-Ernzerh variant [15] of Generalized Gradient Approximation (GGA) and Local (Spin) Density Approximation (L(S)DA) [16] were used for the exchange-correlation potential and they provided very similar results. Similarity of L(S)DA and GGA in analyzing the electronic structures of $\mathrm{MoS}_{2}$ and silicene is also observed in [17]. Hence, we show only results corresponding to GGA. A double- $\zeta$ polarized basis set was used for expanding electronic density. 
$8 \times 16 \times 1 \mathrm{~K}$-points were sampled in the Brillouin zone (BZ) of the side-contact region, while the end-contact BZ was meshed by $8 \times 8 \times 1$ K-points. The density mesh cut-off was 200 Rydberg and the maximum force was $=0.05 \mathrm{eV} / \AA$ for geometry optimizations.

\section{Metal-TMD Contact Evaluation}

Three major criteria are analyzed to evaluate the efficiency of electron injection in contacts as shown in Fig. 2 (d). The electron orbital overlaps (Fig. 2 (e)) are evaluated by optimized geometry (atomic positions), PDOS and electron density calculated by DFT. Schottky and tunnel barriers (Fig. 2 (f)) can be inferred from the PDOS and the effective potential calculated from DFT, respectively.

Optimized Geometries: Configurations with shortest physical separations (defined in Fig. 3 (a) and Fig. 4 (a)) can maximize the overlap between the electron orbitals of the metal and TMD. Optimized geometries (structures with minimum total energy, which reflects the nature of interfaces) are shown in Figs. 3 and 4. Physical separations between the metal and TMD atoms are calculated and listed in Table I. For side-contacts, among high-WF metals (Pd, $\mathrm{Au})$, $\mathrm{Pd}$ gives the smallest physical separations. In low-WF metals (Ti, In), Ti gives the smallest physical separations. Hence, Pd (Ti) allows for stronger orbital overlaps compared with $\mathrm{Au}$ (In).

For all the end-contacts, the physical separations are small and covalent bonds are formed between the metal and TMD. Hence, for a fixed number of TMD atoms contacted by metal, end-contacted configuration is better than the side-contacted configuration.

Partial Density of States (PDOS): PDOS (DOS on specified atoms and orbitals) of side-contacts are shown in Fig. 5 (b)-(e) and (g)-(j), and compared with TMD without contacts (Fig. 5 (a) and (f)). For $\mathrm{MoS}_{2}$ side-contacts, high-WF metals (Au and Pd) form very high SBs to both the conduction band $\left(E_{c}\right)$ and valence band $\left(E_{v}\right)$. Hence, high-WF metals are less suitable for $\mathrm{MoS}_{2}$ side-contact. For low-WF metals, $\mathrm{MoS}_{2}$ can be doped n-type by In or Ti. In-MoS gives an n-type $\mathrm{SB}\left(\Phi_{B N}=E_{c}-E_{F}\right)$ while Ti-MoS $\mathrm{M}_{2}$ is almost an ohmic contact with the highest PDOS near $E_{F}$. For ohmic contacts, a high PDOS near Fermi level $\left(E_{F}\right)$ forms delocalized states with low effective electron mass allowing more electrons to be transferred between the metal and the TMD layer [8].

$\mathrm{WSe}_{2}$ shows p-type PDOS with $\mathrm{Au}$ or Pd side-contacts and is almost intrinsic with In and Ti side-contacts because the $E_{F}$ 's of the In(Ti)-WSe $\mathrm{W}_{2}$ side-contacts lie in the band gap. Although Au and Pd have similar WFs, Pd gives nearly ohmic contact with much higher PDOS near $E_{F}$ than Au indicating stronger orbital overlap than Au. Thus, it is possible to achieve high quality p-type contact on $\mathrm{WSe}_{2}$ with Pd in agreement with recent experimental results [5].

PDOS of end-contacts are shown in Fig. 6. PDOS near $E_{F}$ for all the end-contacts are much higher than that of side-contacts indicating that end-contacted configurations have higher capability of doping the TMDs and can thereby decrease the contact resistance.

Electron Densities: High electron density at the interface indicates a strong overlap of electron orbitals and allows sufficient injection of charge into the TMD layer [8]. Interface electron densities of side-contacts are shown in Fig. 7 and Fig. 8. For sidecontacts, Pd and Ti have higher electron density near the interfaces than $\mathrm{Au}$ and In, implying that $\mathrm{Pd}$ and $\mathrm{Ti}$ have possibility to achieve lower contact resistance.

For end-contacts, electron densities at the interfaces are significantly increased compared with those in side-contacts, as shown in Fig. 8. This is because of the overlap of orbits between metal and TMD atoms. Therefore, the orbital overlap is an advantage of end-contact configurations compared with sidecontacts. Au- $\mathrm{MoS}_{2}$ and $\mathrm{In}-\mathrm{WSe}_{2}$ end-contacts have $2 \mathrm{X}$ interface electron densities compared with that of their side-contacts (Fig. 8 (a)), indicating a decrease in resistance by changing side-contacts to combined-contacts (Fig. 2 (c)). Low contact resistance of combinedcontacted configurations has been demonstrated by experimental results in [3] for $\mathrm{Au}-\mathrm{MoS}_{2}$ and in [11] for In-WSe ${ }_{2}$. Furthermore, the electron localization functions (ELF--a function of the 3Dcoordinates, which is large in the regions where orbitals localize [18 ]) for $\mathrm{Au}-\mathrm{MoS}_{2}$ side- and end-contacts are also shown in Fig. 9. The ELF at the metal-TMD interface is much higher in end-contacts than in side-contacts, indicating that overlap of electron orbitals between metal and TMD atoms is more efficient in end-contacted configurations.

Effective Potential: The effective potential (EP) of an electron represents its interaction with other electrons and the external electrostatic field. The tunnel barrier is the peak of the EP at the interface. A narrow and low tunnel barrier at the metal-TMD interface (Fig. 2 (f)) can increase the current injection efficiency. To evaluate the tunnel barriers, EPs are calculated and shown in Fig. 10 and Fig. 11. For side-contacts, the potential barriers are indicated by red dashed circles in Fig. 10. As shown in Fig. 10, in Ti-TMD and Pd-TMD side-contacts, there is nearly no barrier at the interface while Au-TMD and In-TMD contacts have much higher and wider barriers. Hence, Ti-TMD and Pd-TMD side-contacts have higher electron injection efficiency than Au-TMD and In-TMD sidecontacts. For end-contacts, as shown in Fig. 11, the EPs along $z$ are smoother and with much smaller tunnel barriers compared with those for side-contacts (e.g., Au- $\mathrm{MoS}_{2}$ side-contact $(0.31 \mathrm{eV})$ vs. end-contact $(0.14 \mathrm{eV})$ ), allowing higher electron injection efficiency.

\section{Summary}

Using DFT, optimized geometries, PDOS, electron densities and tunnel barriers of metal-TMD contacts are calculated. We find that from the point of view of the electron injection efficiency, Ti is the best side-contact metal for monolayer intrinsic $\mathrm{MoS}_{2}$ and is an attractive n-type contact metal. Pd is the best side-contact metal for monolayer intrinsic $\mathrm{WSe}_{2}$ and forms p-type contact. It is also shown that end-contacted configurations can improve the contact by lowering physical separation and tunnel barrier as well as increasing the PDOS and electron density. With the right metal and certain contact area, in order to achieve the lowest contact resistance, it is desirable to combine end-contact with side-contact for both monolayer and multilayer TMDs. On the other hand, it is possible to increase the end-contact 'length', for lower contact resistance, by cutting TMD edges into 'jagged' edges. The results obtained in this study not only reveal the types of metals and configurations that can be employed for achieving low contact resistance with TMDs but also highlight that the properties of contacts cannot be intuitively predicted by solely considering WF values, (e.g., Au vs. Pd and In vs. Ti). Moreover, the significance of the developed framework is apparent for a broad range of 2D semiconductors. By combining our framework and transport simulations, quantitative values of contact resistances can be calculated.

\section{REFERENCES}

[1] K. S. Novoselov, et al., Science 306, 666 (2004). [2] K. S. Novoselov, et al., Nature Materials, 6, 183-191 (2007). [3] B. Radisavljevic, et al., Nature Nanotechnology, 6, 147-150 (2011). [4] Y. Yoon, et al., Nano Lett., 11, 9, 3768-3773 (2011). [5] H. Fang, et al., Nano Lett., 12, 7, 3788-3792 (2012). [6] P. Vogt, et al., Phys. Rev. Lett., 108, 155501 (2012). [7] G.-H. Lee, et al., Appl. Phys. Lett., 99, 243114 (2011). [8] I. Popov, et al., Phys. Rev. Lett., 108, 156802 (2012). [9] CRC Handbook of Chemistry and Physics, 12-114 (2008). [10] P. Bhattacharya, Semiconductor Optoelectronic Devices, 2nd Edition, Prentice Hall (1997). [11] W. Liu, K. Banerjee, et al., to be published. [12] S. R. Kumar, et al., Indian J. of Pure \& Appl. Phys., 46, 198203 (2008). [13] Y. Matsuda, et al., J. Phys. Chem. C, 114, 17845-17850 (2010). [14] Atomistix ToolKit v.12.2.2, QuantumWise A/S. [15] J. P. Perdew, et al., Phys. Rev. Lett., 77, 3865 (1996). [16] J. P. Perdew, et al., Phys.Rev.B, 23, 5048 (1981). [17] S. Lebègue, et al., Phys. Rev. B, 79, 115409 (2009). [18] A.D. Becke, et al., J. Chem. Phys., 92, 5397 (1990). 


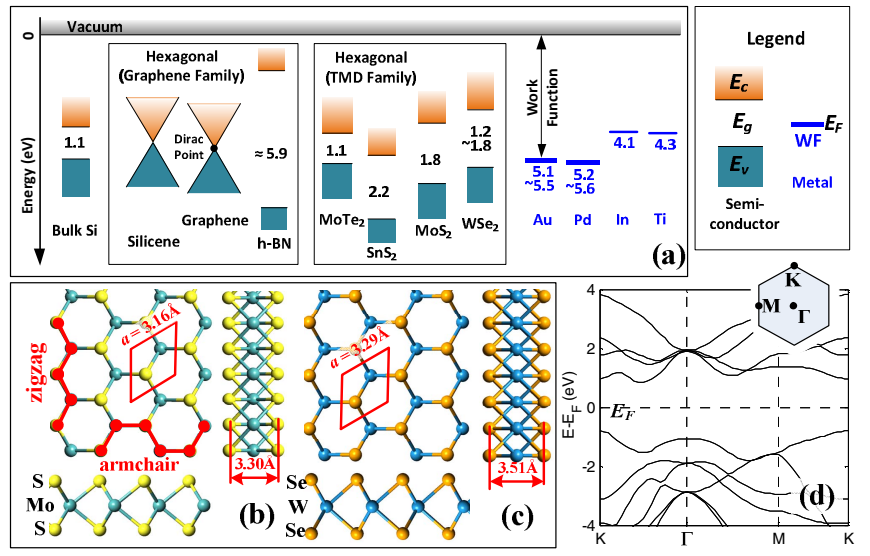

Fig. 1: (a) Band diagrams of some 2D materials and contact metals. $E_{c}, E_{v}$ and $E_{g}$ are conduction band, valence band and band gap, respectively. Metal work functions (WFs) are marked under their Fermi levels $\left(E_{F}\right)$. Silicene is the graphene equivalent for silicon, is synthesized on $\operatorname{Ag}(111)$ surface and has similar electronic dispersion and Dirac points [6]. Hexagonal boron nitride (h-BN) has a large band gap $(5.2 \mathrm{eV}-5.9 \mathrm{eV})$ and hence, it can be used as an extremely thin dielectric layer with dielectric strength $=7.94 \mathrm{MV} / \mathrm{cm}$ [7]. Three views of lattice structures of (b) $\mathrm{MoS}_{2}$ and (c) $\mathrm{WSe}_{2}$. Red rhombuses represent primitive unit cells and $a$ is the lattice constant. Chirality is shown in (b) by armchair edge and zigzag edge. Lattice structures are relaxed using DFT with $8 \times 8 \times 1 \mathrm{~K}$-points sampled in the Brillouin-zone (BZ). (d) Energy dispersions of $\mathrm{MoS}_{2}$. Inset figure in (d) shows the $1^{\text {st }} \mathrm{BZ}$ of TMDs.

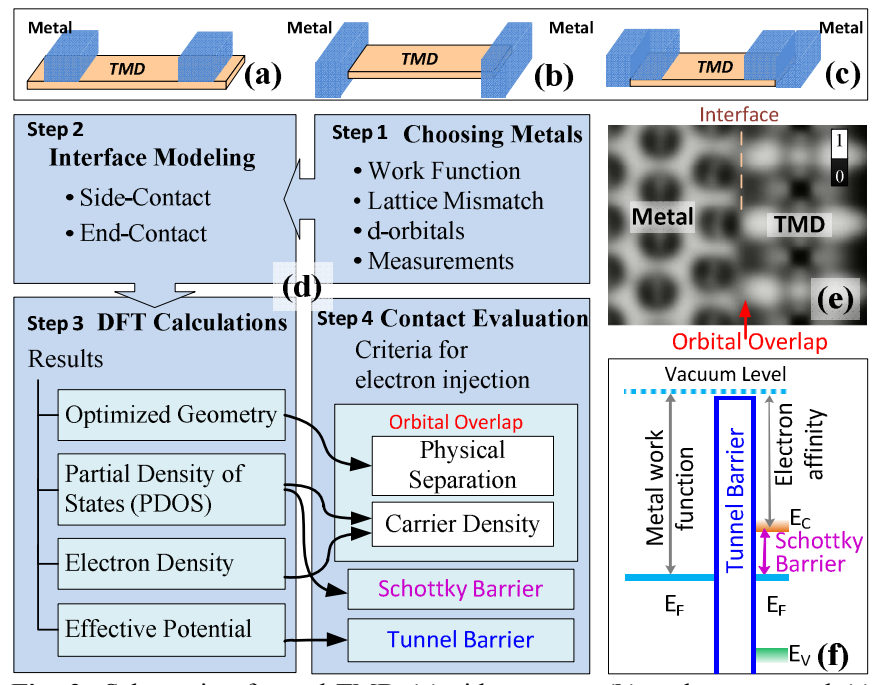

Fig. 2: Schematic of metal-TMD (a) side-contact, (b) end-contact and (c) combined-contact. (d) Flowchart of the framework for metal-TMD contact modeling and simulation. First, metals are chosen based on work functions, lattice mismatches, etc.; In the $2^{\text {nd }}$ step, models for side- and end- contact interface geometries are built; then in the $3^{\text {rd }}$ step, numerical DFT calculations are performed to find out the optimized geometries (defined in the caption of Fig. 3), partial density of states (PDOS), electron density and effective potential in contacts. In the $4^{\text {th }}$ step, orbital overlap (shown in (e)), Schottky barrier and tunnel barrier (shown in (f)) are analyzed using DFT results to evaluate the contacts. (e) Electron probability plot showing orbital overlaps between metal (Ti) and TMD $\left(\mathrm{MoS}_{2}\right)$. (f) Band diagram of an ntype side-contact.

Table I: Physical separations of metal and TMD at the contacts ( $=$ the $z$-component of core-to-core distance between the nearest metalchalcogenide atomic pair as shown in Fig. 3 (a) and Fig. 4 (a)).

\begin{tabular}{|l|l|}
\hline Side-contacts physical separation $(\AA)$ & End-contacts physical separation $(\AA)$ \\
\hline
\end{tabular}

\begin{tabular}{|c|c|c|c|c|c|c|c|c|c|}
\hline TMD & $\mathrm{Au}$ & $\mathrm{Pd}$ & In & $\mathrm{Ti}$ & TMD & $\mathrm{Au}$ & $\mathrm{Pd}$ & In & $\mathrm{Ti}$ \\
\hline $\mathrm{MoS}_{2}$ & 2.93 & 2.33 & 2.42 & 2.01 & $\mathrm{MoS}_{2}$ & 1.70 & 1.72 & 1.38 & 0.84 \\
\hline $\mathrm{WSe}_{2}$ & 3.03 & 2.55 & 3.10 & 2.52 & $\mathrm{WSe}_{2}$ & 2.18 & 1.89 & 1.54 & 1.32 \\
\hline
\end{tabular}
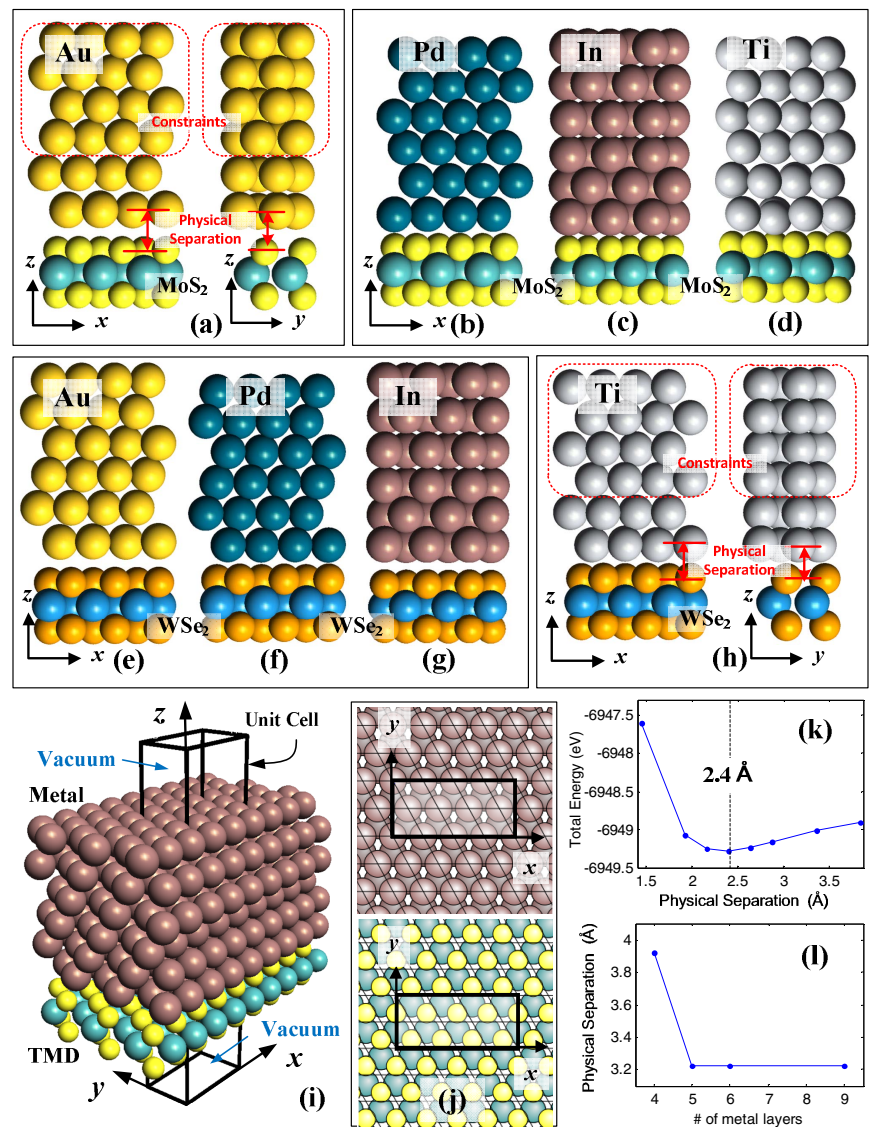

Fig. 3: Optimized geometries of side-contacts (optimized geometry is defined as configurations with the lowest total energy. This is where the structure is most stable and most likely to be found in nature): (a) Au-MoS (b) $\mathrm{Pd}_{-}-\mathrm{MoS}_{2}$; (c) In--MoS $;$; (d) Ti-MoS $;$; (e) $\mathrm{Au}-\mathrm{WSe}_{2}$; (f) $\mathrm{Pd}-\mathrm{WSe}_{2}$; (g) In$\mathrm{WSe}_{2}$; and (h) $\mathrm{Ti}^{-} \mathrm{WSe}_{2}$. Radius of the atomic spheres shown in (a)-(h) are fixed to the covalent radius, which is a measure of atomic size that forms part of one covalent bond. Hence, the overlap of atomic spheres indicates the formation of covalent bonds (e.g., Ti-S bond in (d)). (i) 3D view and (j) top view of In- $\mathrm{MoS}_{2}$ side-contact region showing the periodicity. Vacuum region in $z$-direction is used to separate periodic cells in $z$-direction. (k) Total energy plotted as a function of the separation for $\mathrm{In}-\mathrm{MoS}_{2}$ side-contact. It is clear that the total energy reaches the lowest value at the separation $=2.42 \AA$ (Table I) in the optimized geometry. (l) Metal-TMD physical separation plotted as a funtion of number of metal layers considered in simulation (Al$\mathrm{WSe}_{2}$ side-contact), showing that 6 layers are sufficient for accurate analysis.

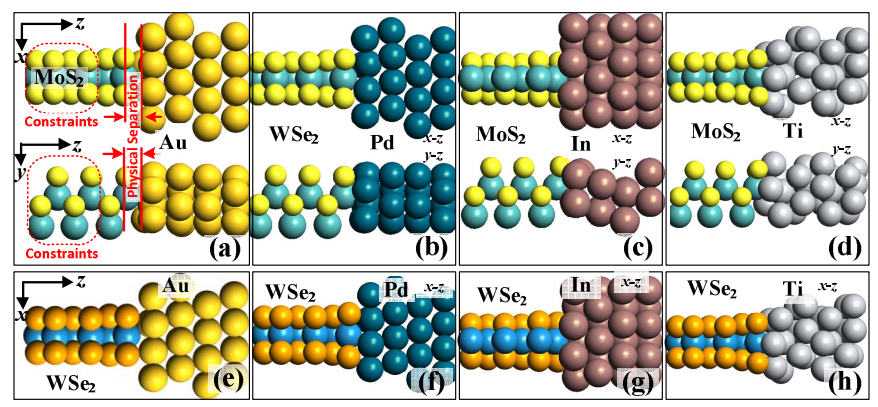

Fig. 4: Optimized geometries of end-contacts: (a) $\mathrm{Au}-\mathrm{MoS}_{2}$; (b) $\mathrm{Pd}-\mathrm{MoS}_{2}$; (c) In- $\mathrm{MoS}_{2}$; (d) $\mathrm{Ti}_{-}-\mathrm{MoS}_{2}$; (e) $\mathrm{Au}-\mathrm{WSe}_{2}$; (f) $\mathrm{Pd}-\mathrm{WSe}_{2}$; (g) In-WSe 2 and (h) Ti$\mathrm{WSe}_{2}$. Both $x-z$ views (upper figures) and $y-z$ views (lower figures) are shown in (a)-(d) while only $x-z$ views are shown in (e)-(h). Physical separations in end-contacts (Table I) are much smaller than those for sidecontacts in Fig. 3 due to covalent bonds formed between TMD and metal. The overlap of atomic spheres exists in every end-contact (in (a)-(h)), indicating the formation of covalent bonds. 


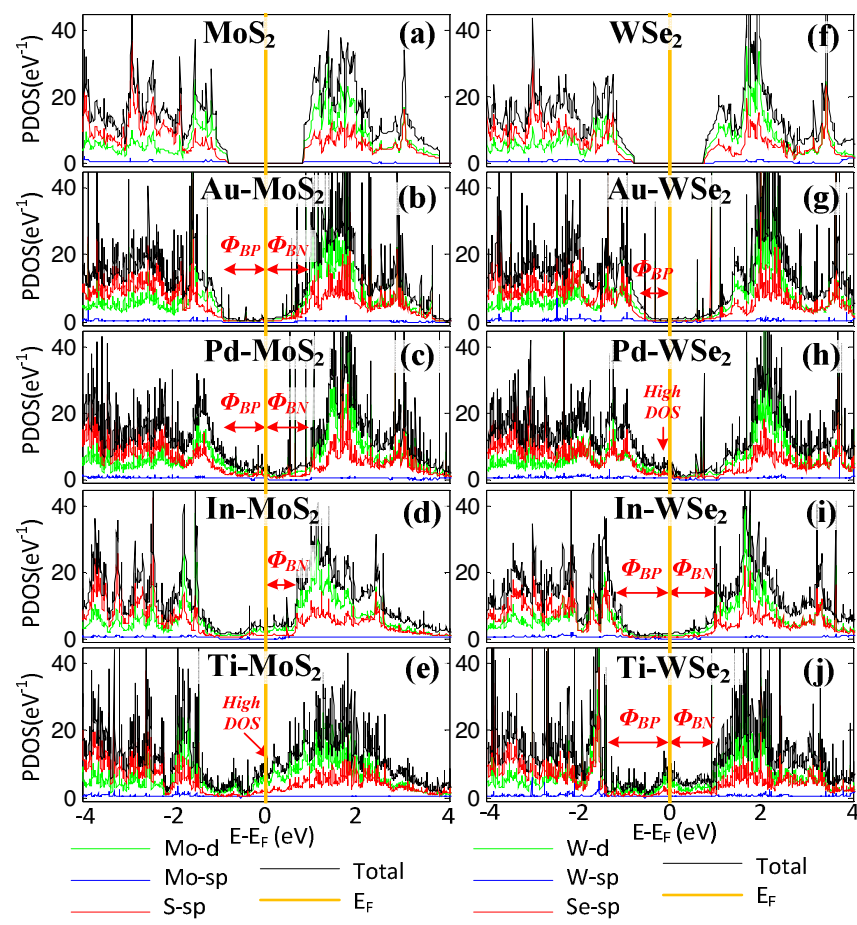

Fig. 5: Partial density of states (PDOS) (DOS on specified atoms and orbitals, for example, Mo-d (d-orbital on Mo)) of side-contacts: (a) only $\mathrm{MoS}_{2}$; (b) Au-MoS $\mathrm{Mos}_{2}$ (c) Pd-MoS $;$; (d) In-MoS $;$; (e) Ti-MoS $2 . \Phi_{B N}$ and $\Phi_{B P}$ are n- and ptype Schottky barriers, respectively. Ti-MoS $\mathrm{S}_{2}$ forms ohmic contact with highest PDOS near Fermi level $\left(E_{F}\right)$. Hence, Ti has the potential to be the best metal for $\mathrm{MoS}_{2}$ side-contact. PDOS of (f) only $\mathrm{WSe}_{2} ;$ (g) Au-WSe $;$; (h) $\mathrm{Pd}-\mathrm{WSe}_{2}$; (i) $\mathrm{In}-\mathrm{WSe}_{2}$; and (j) $\mathrm{Ti}-\mathrm{WSe}_{2}$. In and Ti form Schottky contacts with intrinsic $\mathrm{WSe}_{2}$. Au-WSe $\mathrm{W}_{2}$ and $\mathrm{Pd}-\mathrm{WSe}_{2}$ show similar PDOS except that $\mathrm{Pd}-\mathrm{WSe}_{2}$ has much higher PDOS near $E_{F}$. Thus, $\mathrm{Pd}-\mathrm{WSe}_{2}$ is nearly an ohmic contact, which explains experimetal data in [5].

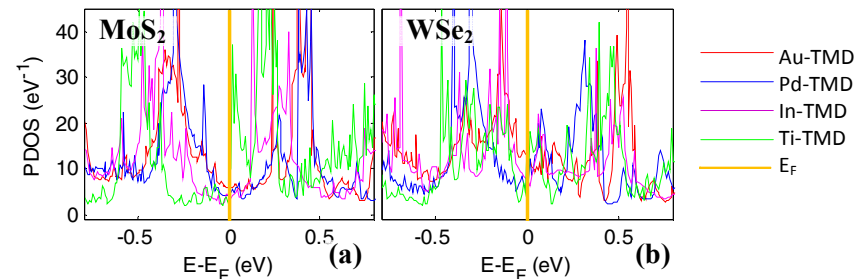

Fig. 6: PDOS of TMD near Fermi level $\left(E_{F}\right)$ of end-contacts: (a) $\mathrm{MoS}_{2}$ and (b) $\mathrm{WSe}_{2}$. TMDs are metalic due to doping effects of end-contact metal. All the end-contacts have higher PDOS near $E_{F}$ than the side-contacts in Fig. 5 indicating that end-contacts have lower contact resistance than side-contacts.

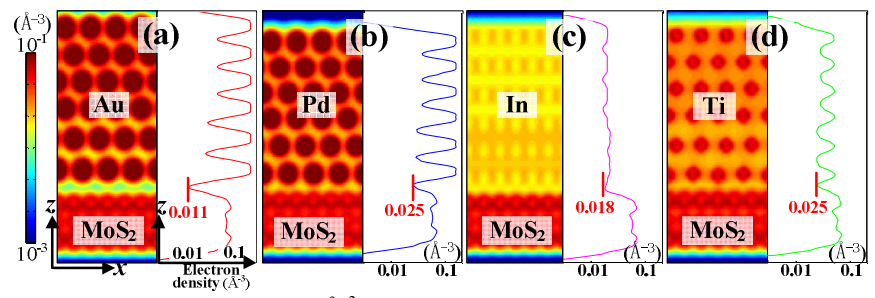

Fig. 7: Electron densities $\left(\AA^{-3}\right)$ of metal- $\mathrm{MoS}_{2}$ side-contacts: (a) $\mathrm{Au}-\mathrm{MoS}_{2}$; (b) $\mathrm{Pd}-\mathrm{MoS}_{2}$; (c) In-MoS 2 and (d) Ti-MoS 2 . In each sub-figure ((a)-(d)), the left contour shows average density along $y$ projected on $x-z$ plane; the right plot shows average value in $x-y$ planes normal to $z$-axis. Color bar on the left is common for all contours. Red numbers on curves indicate the lowest electron densities at the interfaces. Among low-WF metals, Ti has higher electron density at the interface while Pd has higher interface electron density among high-WF metals, indicating that $\mathrm{Ti}$ and Pd may form better side-contacts.

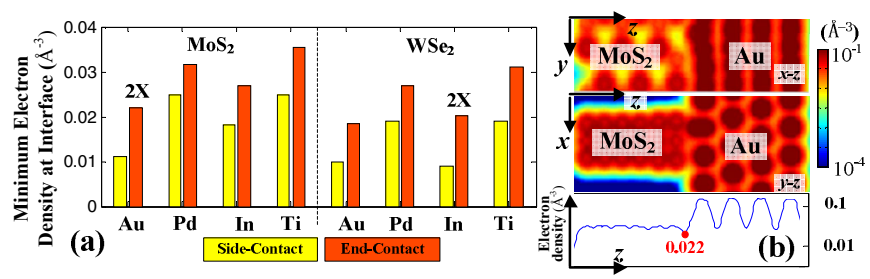

Fig. 8: (a) Minimum of average electron density values in $x-y$ plane at the interface for all side- and end-contacts. Ti and Pd may form better sidecontacts due to higher interface electron density. Electron densities are significantly increased for end-contacts. (b) Electron densities of $\mathrm{Au}-\mathrm{MoS}_{2}$ end-contact. The three figures (from top to bottom) are averages on $y$-z plane, $x-z$ plane and along $z$-axis. Red number in botom figure of (b) indicates the lowest electron density at the interface.
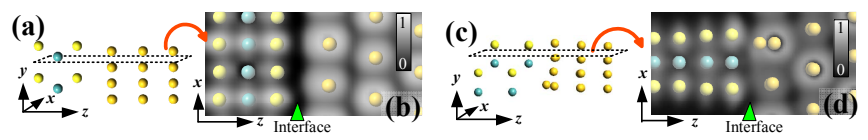

Fig. 9: The cleaved $x-z$ planes (dashed rectangles in (a) and (c)) used to show electron localization functions (ELF) for side-contact and end-contact in $\mathrm{Au}-$ $\mathrm{MoS}_{2}$. ELF plots on the cleaved $x-z$ plane in $\mathrm{Au}-\mathrm{MoS}_{2}$ (b) side-contact; (d) end-contact. Half-transparents dots indicate positions of atoms. High ELF indicates high probability of finding an electron. For end-contact, $\mathrm{Au}-\mathrm{MoS}_{2}$ has overlapping electron orbitals resulting in the increasing of electron density as shown in Fig. 8 (a). Thus, the contact resistance is reduced by using end-contacts.
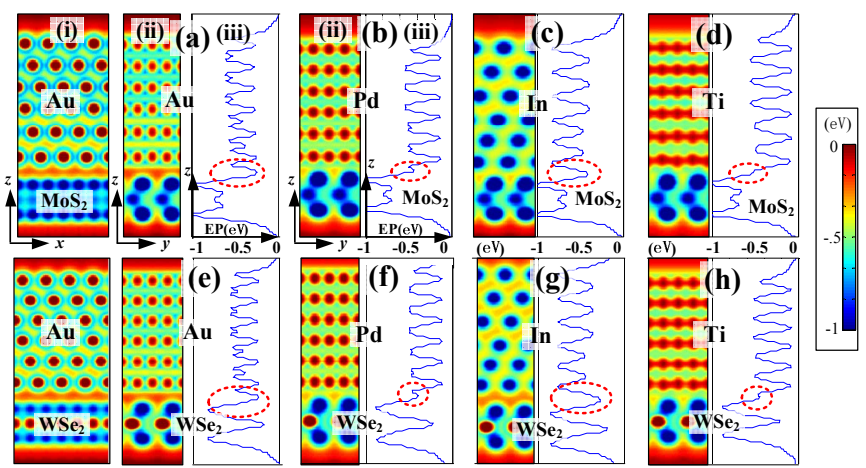

Fig. 10: Effective potential (EP, which reflects the interaction of an electron with all other electrons and the external potential) of side-contacts: (a) Au-

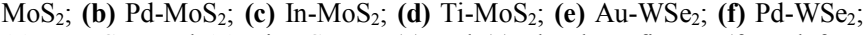
(g) In-WSe ${ }_{2}$; and (h) $\mathrm{Ti}_{-}-\mathrm{WSe}_{2}$. In (a) and (e), the three figures (from left to right) are (i) average effective potential along $y$ projected on $x$-z plane; (ii) average value along $x$ projected on $y-z$ plane; and (iii) average value in $x-y$ planes normal to $z$-axis. In (b)-(d) and (f)-(h), the first plot (i) in (a) and (e) is omitted. Color bar on the right is shared by all the contours. Red dashed circles indicate the tunnel barriers at the interfaces. Ti-TMD and Pd-TMD side-contacts have very low tunnel barriers indicating low contact resistance.

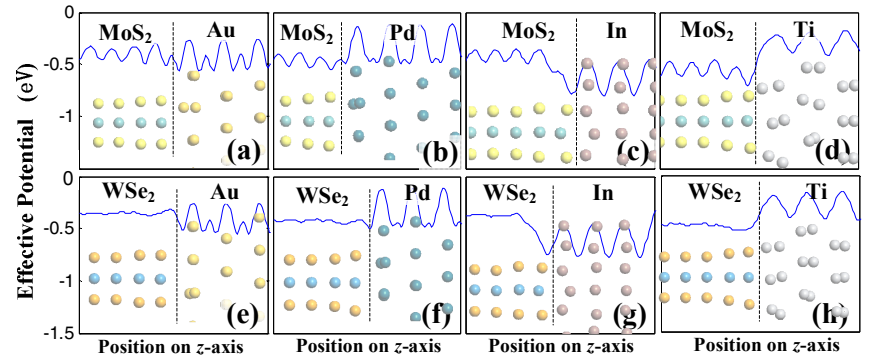

Fig. 11: Average effective potential of end-contacts along $z$-axis: (a) Au$\mathrm{MoS}_{2}$; (b) $\mathrm{Pd}-\mathrm{MoS}_{2}$; (c) In- $\mathrm{MoS}_{2}$; (d) Ti-MoS ; (e) $\mathrm{Au}-\mathrm{WSe}_{2}$; (f) $\mathrm{Pd}-\mathrm{WSe}_{2}$; (g) In-WSe $\mathrm{W}_{2}$; and (h) $\mathrm{Ti}-\mathrm{WSe}_{2}$. Half-transparent dots indicate positions of atoms. Tunnel barriers are much smaller in end-contacts than in side-contacts. Thus, end-contacts have the potential to allow higher current injection than side-contacts. 